\title{
Positive aspects of digitalization in radiation therapy
}

\section{Opinion}

As a health professional, serving the oncology community of Panama, I have seen through my almost 25 years of service, how important has been the advancement of technology, which has allowed the creation of new treatment techniques, and therefore, he has led increasingly to preserve healthy tissue and reduce toxicity in patients who are undergoing radiotherapy.

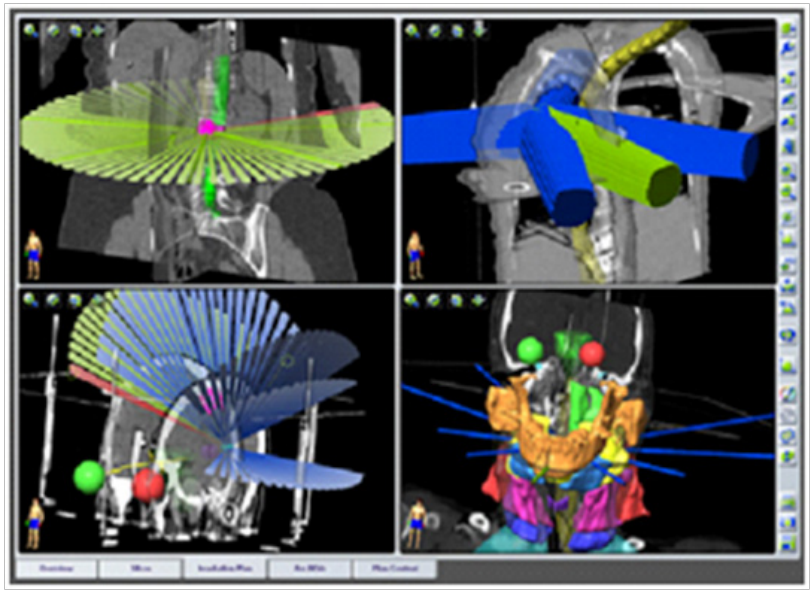

Radiation therapy is not more than the use of high-energy radiation to treat cancer. Approximately $60 \%$ of cancer patients receive radiation therapy at some point during the course of their treatments. The process involves a series of steps involving simulation and planning before treatment.

In 1993, the year I began my career as a Technical Assistant Radiotherapy, National Cancer Institute had 2 teams Cobalt 60 for the treatment of patients and a section of low dose rate brachytherapy with Cesium 137. Sources then, privately, there was only a team of Cobalt 60 in the San Fernando Hospital Clinic.

These teams Cobalt had a console, which, despite having digital timers, the method of introducing parameters such as treatment time was manually. There was a network connection that allowed transfer the parameters from the planning system to the treatment team.

For the simulation process, images were taken with a team of conventional x-rays and proceeded to score on the board acquired areas to protect, and then make blocks of custom lead, or, standard blocks, which were accessories of the respective teams.

Also during the planning process, calculations were made in 2D medium deep (two-dimensional) and plane. There was no possibility in our country, at that time, making plans that would allow preserving the surrounding healthy tissues to areas of interest, so margins were relatively large.

The treatments were performed with fixed fields, AP-PA and orthogonal. This caused that obese patients present severe radiodermitis; patients with treatments in the pelvic area, showed high toxicity in rectum and bladder, and many other conditions in patients

\author{
Volume 9 Issue I - 2018
}

Jasmina Alexander

Radiology and Radiation Therapy Technician, USA

Correspondence: Jasmina Alexander, Novalis Treatment Center, Panama City, Punta Paciาfica John Hopkins Hospital, Basement 2, Panama, USA, Tel 5073963162. Email jilexa@gmail.com

Received: December 14, 2017 | Published: January 31, 2018

of different diagnoses, which were almost impossible to avoid with techniques which had allowed or equipment that time.

For 1999, a private center acquired a linear accelerator with an analog-digital console; Similarly, the virtual simulation was introduced in our country, with the use of CT images, which were used for treatment planning, which allowed to venture into what was the $3 \mathrm{D}$ planning. Even then, Panama was 20 years overdue with respect to equipment and techniques and treatment planning worldwide in external beam radiotherapy. However, with the implementation of $3 \mathrm{D}$ planning and support of digital images obtained from the scan, it was possible to decrease toxicity in patients considerably. Four years later, the National Cancer Institute acquired 3 linear accelerators that allow the planning system to incorporate $3 \mathrm{D}$ in the public sector. These accelerators were the first fully digital console equipment and a network system that allowed the transfer of all patient parameters (digital file). This embodiment, be passed to a safety barrier allowing greatly reduce the probability of error when manually enter parameters such as time of treatment, among others.

Similarly, pioneering IMRT national center, replaced the low dose rate brachytherapy sources Cesium 137, by using high rate brachytherapy sources iridium 192. This means an automated system allowing direct sources and administered to the tumor bed the dose required in minutes or hours.

Later, in 2012, at the level of the private sector, it starts in Panama treatments IMRT (Intensity Modulated Radiation Therapy), which allow modulating the radiation beam, thus reducing, much more, toxicity in the organs at risk. For this treatment technique teams already have verification system digital images, which allow the necessary corrections just before starting each session. 


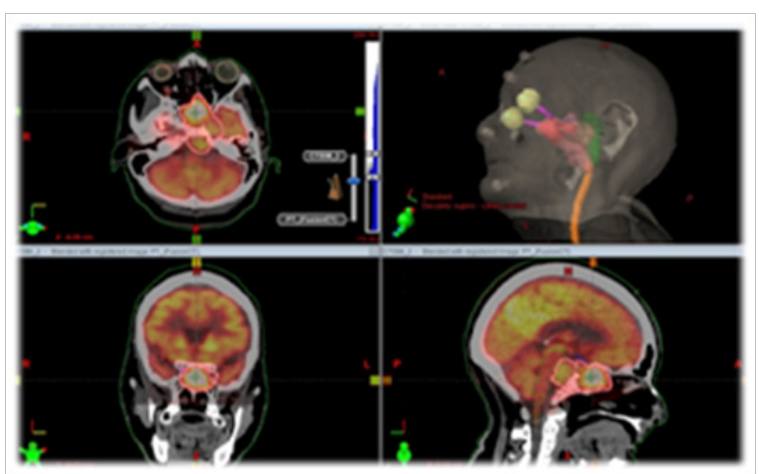

Already in 2013, I had the opportunity to be part of the history of radiotherapy in Panama, operating the first Linear Accelerator True Beam STx Central, which is fully digitized and high-tech addition to using treatments allowed by the technique IMRT, IGRT to perform (Image Guided Radiation Therapy). With this treatment technique can correct the position of the patient performing a Cone Beam CT $(\mathrm{CBCT})$ to the patient, once positioned on the treatment table, what is also known as adaptive radiation therapy, and who daily adjust device parameters to the internal movements of the patient's organs, thereby avoiding irradiating healthy tissue areas or because of the movements of the patient and therefore their bodies.

This can also be achieved today using positioning systems radiosurgery (type of radiation therapy where high radiation doses are administered once or up sessions only), as Exac Trac Brain Lab system, where we can see in real time the position of the lesion and thus administer treatment.

Similarly, for the simulation phase, both teams simulation, planning and treatment, have a "protocol language" that allows fusion of images. Thus, digital images obtained in the virtual simulator (CT) can be fused with MR imaging or PET-CT for treatment planning; because, by having the same origin DICOM3, image fusion is performed by planners simply and quickly without having to resort to common anatomical points of the two scans.

So thanks to all these positive aspects of digitization in the passing of the years of performance of my profession as Technologist in Radiotherapy and Radiology and Medical Imaging, I could see how our country has evolved technological level; and also, I have had the opportunity to demonstrate, through practice, the great importance of digitization in my work area.

As for the benefits, I can assure you that they have not only resulted around the patient, who today, through the variety of equipment and techniques digitized, they can enjoy a better quality of life through reduced side effects caused by radiation, due to the lower toxicity in organs at risk; but also, they have fallen around the professionals responsible for prescribing, planning and administration of treatments, since we see the lesions to be treated in its entirety and can monitor in real time the work done, thanks to technology 4D here to stay.

\section{Conflicts of interest}

None.

\section{Acknowledgements}

None.

\section{Funding}

None. 